\title{
Nano-Dielectric Resonator Antenna Reflectarray/Transmittarray for Terahertz Applications
}

\author{
Hend A. Malhat*, Nermeen A. Eltresy, Saber H. Zainud-Deen, and Kamal H. Awadalla \\ Faculty of Electronic Eng., Menoufiya University, Egypt \\ *corresponding author, E-mail: er_honidaleyahoo.com
}

\begin{abstract}
Nanoantennas have been introduced for different applications as solar-cell, medical imaging, and fast data communications. The material properties of good conducting metals introduce plasmonic behavior at terahertz frequencies. The electrical properties of different good conducting metals are modelled using the Drude model and have been investigated for reflectarray/ transmitarray operation. The radiation characteristics of nano-dielectric resonator antenna (NDRA) reflectarray at $633 \mathrm{~nm}$ have been investigated. The unit-cell consists of NDR mounted on the metallic supporting plane. A parametric study for the NDR unit cell dimensions and material has been introduced. The NDR unit-cell with silver supporting plane has been designed and analyzed for reflectarray applications at 633 $\mathrm{nm}$. The radiation characteristics of the NDRA transmitarray has been introduced for $633 \mathrm{~nm}$ applications. The NDR unit-cell consists of two NDRs mounted on the top and bottom of the metallic supporting plane. A comparison between the radiation characteristics of $17 \times 17$ and $21 \times 21$ NDRA transmitarray has been introduced. A compromise between the nano-transmitarray size, maximum gain, and operating bandwidth is applied to terahertz applications. The finite integral technique is used to carry a full wave analysis to design an NDRA reflectarray and an NDRA transmitarray..
\end{abstract}

\section{Introduction}

The radiation characteristics of a conventional radiofrequency (RF) antenna have been presented in detail in [1]. Nanoantenna is a resonant device, which converts the electromagnetic wave into a localized energy at terahertz frequencies [2]. Recently, wide bandwidth nanoantennas have been introduced for faster information exchange. Nanoantennas have many applications including solar cells, on-chip wireless optical communication and biological imaging. Different forms of the microwave antennas such as dipole, monopole, Yagi-Uda, and bow-tie antenna have been investigated at the terahertz frequencies [3-6] which focus on resonant metallic nanostructures. The materials used for nanoantennas fabrication are generally good conducting metals such as gold and silver [7]. The resonant structures of good conducting metals show electromagnetic resonances, when being excited by an incident light, this is called surface plasmon polariton resonances (SPPRs). The electrical properties of these metals are described by the DrudeLorentz model which considers both the free electrons contributions and harmonic oscillator SPPRs contributions [8].

The optical properties of the nanoantenna depend on the size, geometry and material. However, only the high ohmic losses of metals at terahertz frequencies affect the radiation efficiency of nanoantennas [2]. The nanoantenna resonance length is not determined by the free space wavelength, but by the SPP wavelength in the metal [2]. The current distribution on the nanoantenna has a standing wave pattern similar to that of the RF antennas, but with non-uniform spacing between subsequent current lobes. Nanoantenna arrays introduce a superior directivity, field confinement, absorption cross-section and flexibility in beam shaping compared with single nanoantennas. Dielectric resonator antennas (DRA) have many attractive features and applications at microwave frequencies [9]. The DRAs have different shapes as a hemisphere, cylinder, or rectangular and are typically mounted on a metal layer regarded as perfect electric conductor. The DRAs are generally constructed from low-loss high-permittivity dielectric materials (up to $\varepsilon_{\mathrm{r}}=100$ ). To increase the efficiency of resonant nanoantennas the low-loss high-permittivity dielectric materials available at terahertz frequencies are used. At terahertz frequencies, the wave penetrates the metals due to the plasmonic effect and the antenna scaling property is not valid. The radiation characteristics of the DRA at $633 \mathrm{~nm}$ have been investigated in [10]. High-gain microwave antennas have been used in many applications such as radar and satellite communications. The parabolic reflector and phased array antenna have high gain, narrow main lobe and high power capacity, but they suffer some disadvantages as high cost, and large volume for the parabolic reflector, and lossy feed networks in a phased array. Reflectarray antenna combines the advantages of parabolic reflector and phased array and overcomes their disadvantages [11]. The reflectarray antenna consists of a primary source illuminating a planar surface composed of an array of unit-cells. The phase shift of each unit-cell is adjusted to collimate the reflected wave in the desired direction. Reflectarray suffer from feeder blocking effect, so 
it requires an offset feed to avoid blockage losses, which leads to destroying the symmetry of the antenna aperture and increases the angle of incidence of some individual elements [12]. The transmitarray overcomes reflectarray feed blocking problem, and both the reflectarray and transmitarray transform the spherical wave emanating from the feeder into a plane wave. Transmitarray is similar to the reflectarray, but the incident wave is not reflected but passes through the antenna structure as it is collimated into a plane wave on the other side. 9x9 unit-cells elements, nanoreflectarray using single perforated silver sheet at $735 \mathrm{THz}$ has been introduced [13]. A detailed analysis of the radiation characteristics of nano-dielectric resonator antenna single element and the NDRA reflectarray at $633 \mathrm{~nm}$ is investigated in [10]. A circularly polarized graphene transmitarray for terahertz application has been presented in [14].

In this paper, the electrical material properties of metals at terahertz frequencies have been determined. A parametric study of the NDRA unit-cell for reflectarray at $633 \mathrm{~nm}$ has been introduced. The effect of changing the metal material property on the performance of the NDRA unit-cell is investigated. A $21 \times 21$ unit-cell elements NDRA reflectarray has been designed and analyzed using the finite integral technique based on the commercial software CST microwave studio $[15,16]$. A parametric study of an NDRA unit-cell with the silver supporting plane for transmitarray operating at $633 \mathrm{~nm}$ has been proposed. The radiation characteristics of the $21 \times 21$ NDRA transmitarray are determined and compared to a $17 \times 17$ NDRA transmitarray..

\section{Material properties at Terahertz range}

At terahertz frequencies, the behaviour of conventional metals properties behaves in a different way compared to the microwave frequencies [7]. In microwave frequency range the electric field inside the conductors is zero, which leads to perfect reflection from the surface of the metal, as the conductivity of metal is very high. However, at terahertz frequencies, the assumption of perfect conductor metals is not valid and the losses cannot be neglected [8]. The material properties in the terahertz range can be described by a free electron gas moving through a lattice of positive ions. The frequency dependent complex permittivity and the electrical conductivity of metal in the terahertz frequency range is described using the Drude model given by [8]

$$
\begin{gathered}
\varepsilon=\varepsilon_{1}+j \varepsilon_{2}=\varepsilon_{0}\left[1-\frac{\omega_{D}^{2}}{\omega\left(\omega-j \theta_{p}\right)}\right] \\
\sigma=\sigma_{1}+j \sigma_{2}=\varepsilon_{0} \frac{\omega_{D}^{2}}{j \omega+\theta_{p}}
\end{gathered}
$$

where $\varepsilon_{1}$ is the real part of permittivity and is a measure of how much energy from an external field is stored in a material, $\varepsilon_{2}$ is the imaginary part of permittivity (loss factor) and is a measure of how dissipative or lossy a material is to an external field, $\varepsilon_{0}$ is the dielectric constant of vacuum, $\omega$ is the angular frequency of the electromagnetic wave, $\vartheta_{p}$ the angular collision frequency, and $\omega_{p}$ is the electron plasma angular frequency [8]

$$
\omega_{p}=\sqrt{n_{e} q^{2} / \varepsilon_{0} m_{e}} \quad=56.40 \sqrt{n_{e}}
$$

where $n_{e}$ is the free electron density, $m_{e}$ is the electron mass, and $q$ is the charge of the electron. Figure 1 shows the variation of electric permittivity $\varepsilon$, and the conductivity $\sigma$, versus frequency in the terahertz range of gold, copper, silver and aluminum. The electrical permittivity and conductivity of all metals take an exponential variation with frequency. $\varepsilon_{1}$ and $\sigma_{2}$ (the permittivity components of $\varepsilon$ and $\sigma)$ are increased by increasing frequency (negative with reduced magnitude with higher frequency) while $\varepsilon_{2}$ and $\sigma_{1}$ (the conductivity components with $\sigma_{1}$ normally dominating at lower frequencies) are decreased in magnitude by increasing frequency. The skin depth $\delta(\omega)$ represents how deep the electromagnetic wave can penetrate the material surface (1/e of its initial value at the surface) [8]

$$
\delta(\omega)=\frac{2 c \sqrt{\frac{\varepsilon_{1}}{2}+\frac{1}{2} \sqrt{s_{1}^{2}+\varepsilon_{2}^{2}}}}{\omega s_{2}}
$$

where $c$ is the speed of light. The variation of skin- depth versus frequency for different metals is shown in Fig.2. At $474 \mathrm{THz}$, the skin depth is $32 \mathrm{~nm}$ for gold, $28 \mathrm{~nm}$ for copper, $24.5 \mathrm{~nm}$ for silver and $17 \mathrm{~nm}$ for aluminum.

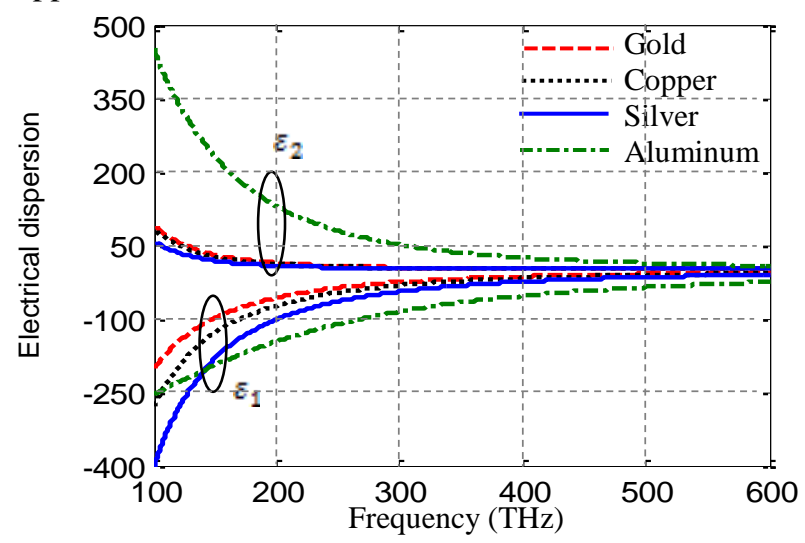

(a) Electrical Dispersion

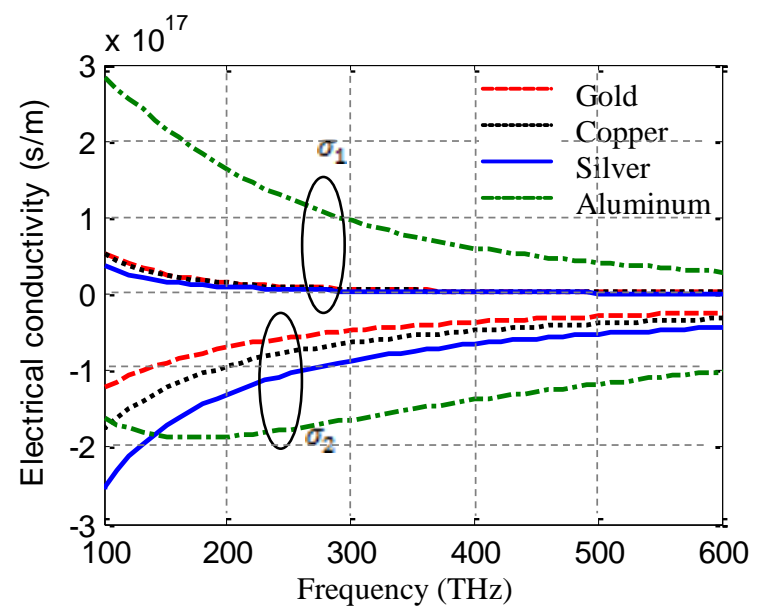

(b) Electrical conductivity

Figure 1 The variations of complex permittivity and electrical conductivity versus frequency for different types of metals. 


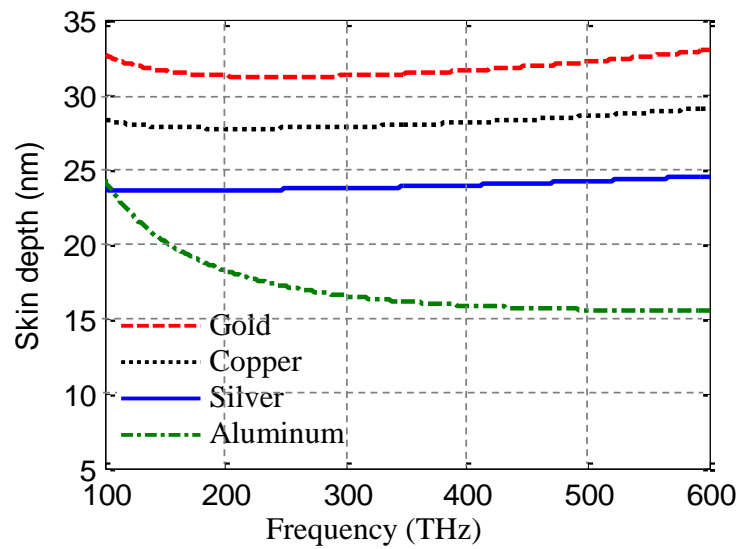

Figure 2 The skin depth variation versus frequency for silver, gold, copper and aluminum metals.

\section{Theory of Reflectarray/transmitarray Antenna}

The reflectarray/transmitarray operation can be seen as a phased array with spatial feed located at $\left(x_{f}, y_{f}, z_{f}\right)$ from the array aperture. For the reflectarray/transmitarray located in $\mathrm{x}-\mathrm{y}$ plane the wave is reflected/transmitted from each unitcell at direction $\left(\theta_{0}, \emptyset_{0}\right)$ suffer from additional phase shift due to the position of the element in the array ( $\left.x_{\text {cijo }} y_{c i j}\right)$ and spacing between the cell element and the feeding horn $d_{i j}$ as shown in Fig.3. To collimate the reflected/transmitted wave at direction $\left(\theta_{0}, \emptyset_{0}\right)$ each unit-cell requires a compensation phase

$$
\varphi_{i j}\left(x_{c i j}, y_{c i j}\right)=k_{0} d_{i j}+\emptyset_{c i j}
$$

where $k_{0}=2 \pi / \lambda$ is the wave number, $d_{i j}$ is the distance from the feeding horn to each unit cell [12]

$$
d_{i j}=\sqrt{\left(x_{c i j}-x_{f}\right)^{2}+\left(y_{c i j}-y_{f}\right)^{2}+z_{f}^{2}}
$$

and $\varphi_{c i j}$ is the phase shift due to the location of the unit-cell in the array

$$
\varphi_{c i j}=-x_{c i j} \sin \theta_{0} \cos \emptyset_{0}-y_{c i j} \sin \theta_{0} \sin \emptyset_{0}
$$

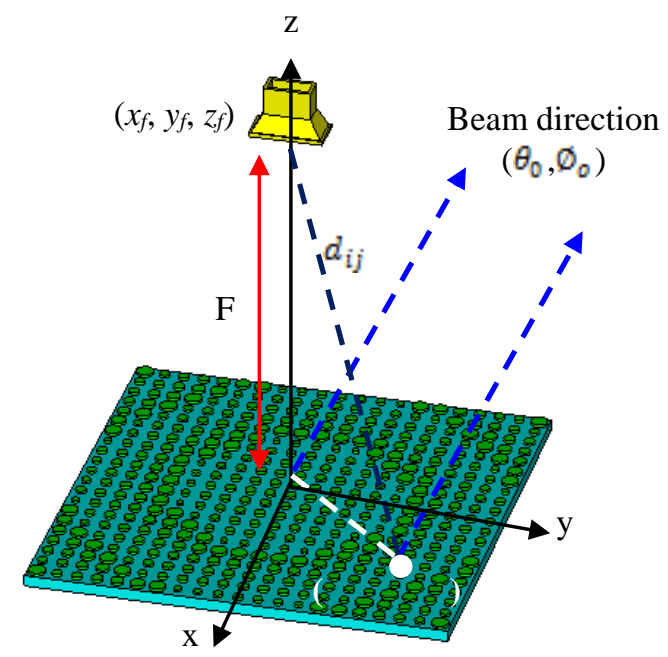

Figure 3 The detailed structure of the reflectarray/ transmitarray configuration.

\section{Design of NDRA reflectarray antenna}

The unit-cell of nano dielectric resonator reflectarray consists of an NDR made of titanium dioxide $\left(\mathrm{TiO}_{2}\right)$ with anisotropic frequency independent dielectric relative permittivity of 8.29 in $\mathrm{x}$ - and $\mathrm{y}$-axis directions and $6.71 \mathrm{in} \mathrm{z}$ axis direction, and estimated loss tangent of 0.001 [10]. The NDR has a cylindrical shape with radius $R$ and height $h_{d}$ placed on a square ground plane with thickness $h$ to operate at $474 \mathrm{THz}$ as shown in Fig. 4a. To calculate the required reflection coefficient phase compensation in each unit cell, the unit cell is put in a waveguide simulator [13]. The perfect electric and magnetic wall boundary conditions are posted onto the sides of the surrounding waveguide, and result in an infinite array. A linearly polarized plane wave was applied as the far-field excitation of the unit cells inside the waveguide simulator and only normal incidence angle is considered. There are several limitations to the infinite array approach. First, all elements of the reflectarray/ transmitarray are identical; this is not the case in the real reflectarray/ transmitarray in which the diameters of the NDR in each cell element must vary according to the required phase compensation. Secondly, the reflectarray/ transmitarray itself is not infinite in extent. Finally, only normal incidence is considered. However, the plane wave has an oblique angle on the real array element, but the phase variation is nearly the same for incidence angles up to $30^{\circ}$ [14]. Different metals are used for ground plane as silver, gold, copper, and aluminum. The properties of the ground plane metals are determined using Eq. (1) at $474 \mathrm{THz}$ and are listed in Table I.
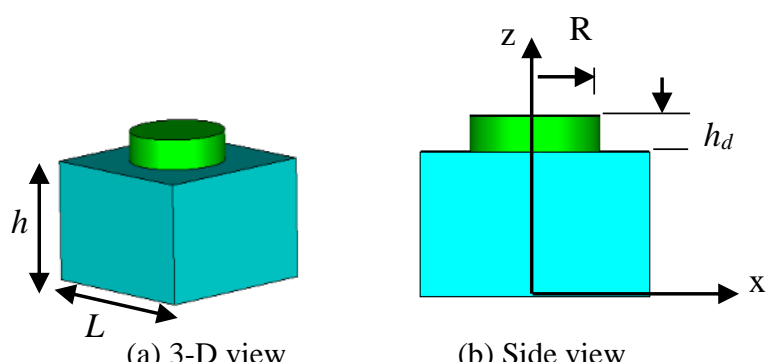

(b) Side view

Figure 4 The detailed structure of the NDR reflectarray unit cell.

The required compensation phase of the reflection coefficient for each unit-cell is achieved by varying the NDRA radius $R$. Figure 5 shows the variation of the reflection coefficient magnitude and phase versus the NDR radius at $474 \mathrm{THz}$ for different ground plane metals. From the losses point of view the gold ground plane has the worst reflection coefficient (i.e. higher losses) variation from -30.4 $\mathrm{dB}$ to $-2.9 \mathrm{~dB}$, while the Silver ground plane gives the best reflection coefficient magnitude (i.e. lower losses) varies from $-5.8 \mathrm{~dB}$ to $-1.7 \mathrm{~dB}$ for the NDR radius varying from 85 $\mathrm{nm}$ to $170 \mathrm{~nm}$. This is because the conductivity of the gold material is higher than that of the silver material at $474 \mathrm{THz}$ as appeared in Fig1.b and the penetration in aluminum ground plate is higher than that of silver Fig. 2. 
Table I. The optical properties of the different types of metals at $474 \mathrm{THz}$ [17]

\begin{tabular}{|l|l|l|l|l|l|}
\hline Metal & $\omega_{p}(\mathrm{rad} / \mathrm{sec})$ & $\mathrm{v}_{p}(\mathrm{rad} / \mathrm{sec})$ & $\varepsilon_{y}$ & $\sigma$ & $\delta(\mathrm{nm})$ \\
\hline Silver & $1.28 \times 10^{16}$ & $9.19 \times 10^{13}$ & $-17.46+\mathrm{j} 0.56$ & $0.169 \times 10^{16}-\mathrm{j} 5.49 \times 10^{16}$ & 24.5 \\
\hline Gold & $0.98 \times 10^{16}$ & $2.8 \times 10^{14}$ & $-9.27+\mathrm{j} 0.95$ & $0.285 \times 10^{16}-\mathrm{j} 3.05 \times 10^{16}$ & 32 \\
\hline Copper & $1.13 \times 10^{16}$ & $3.2 \times 10^{14}$ & $-12.7+\mathrm{j} 0.87$ & $0.26 \times 10^{16}-\mathrm{j} 4.04 \times 10^{16}$ & 28 \\
\hline Aluminum & $2.3 \times 10^{16}$ & $1.04 \times 10^{15}$ & $-40+\mathrm{j} 15.2$ & $4.5 \times 10^{16}-\mathrm{j} 12.2 \times 10^{16}$ & 17 \\
\hline
\end{tabular}

The phase of the reflection coefficient span of variation is $360^{\circ}$ for the silver, $19^{\circ}$ for gold, $215^{\circ}$ for the copper, and $136^{\circ}$ for the aluminum ground plate. The silver material has the best performance for the reflectarray unit-cell with reflection coefficient magnitude variation from $-5.8 \mathrm{~dB}$ to $1.7 \mathrm{~dB}$, and phase variation from 0 to $360^{\circ}$. Figure. 6 shows the effect of changing the silver ground plane thickness $(h)$ of the unit cell on the variation of the reflection coefficient magnitude and phase. By increasing the ground plane thickness $(h)$, the reflection coefficient magnitude is decreased while the reflection coefficient phase variation is increased to achieve $360^{\circ}$.

A compromise between the magnitude and phase of the reflection coefficient has been made. A ground plane of thickness $h=200 \mathrm{~nm}$ has been chosen for which the reflection coefficient magnitude varies from $-1.7 \mathrm{~dB}$ to -5.8 $\mathrm{dB}$ and $360^{\circ}$ phase variations. Silver ground plane with thickness $h=200 \mathrm{~nm}$ has a slower phase variation than $\mathrm{h}=30 \mathrm{~nm}$ which introduces a wider operating bandwidth and has a higher reflection coefficient magnitude in the area of interest of the NDR radius changes from $R=105 \mathrm{~nm}$ to 170 $\mathrm{nm}$. The electric field distribution on the unit-cell of the NDR reflectarray with silver supporting plane $h=200 \mathrm{~nm}, h_{d}$ $=50 \mathrm{~nm}$, and $R=130 \mathrm{~nm}$ is shown in Fig 7. The incident plane wave penetrates the silver supporting plane at a distance equal to the skin depth and reflects back to the source direction. The reflection occurs because the thickness (h) of the silver supporting plane is much bigger than the silver skin depth (about 8.16 $\delta$ ).

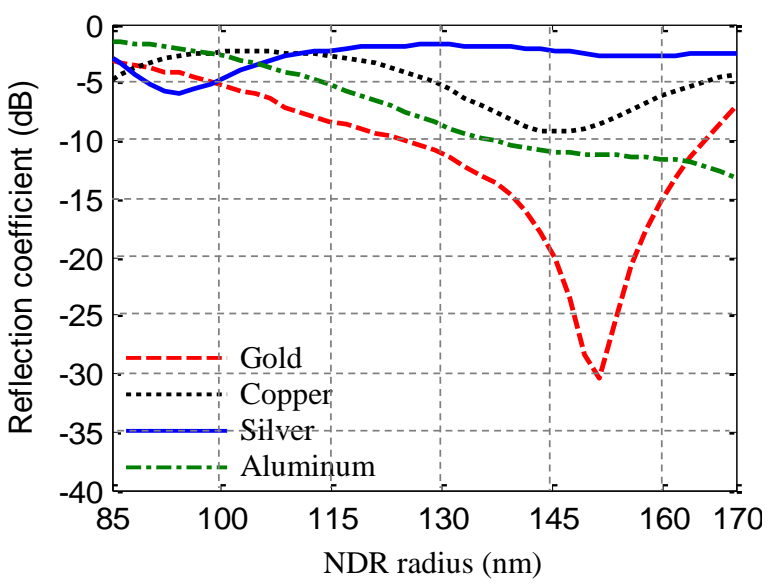

(a) Reflection coefficient magnitude

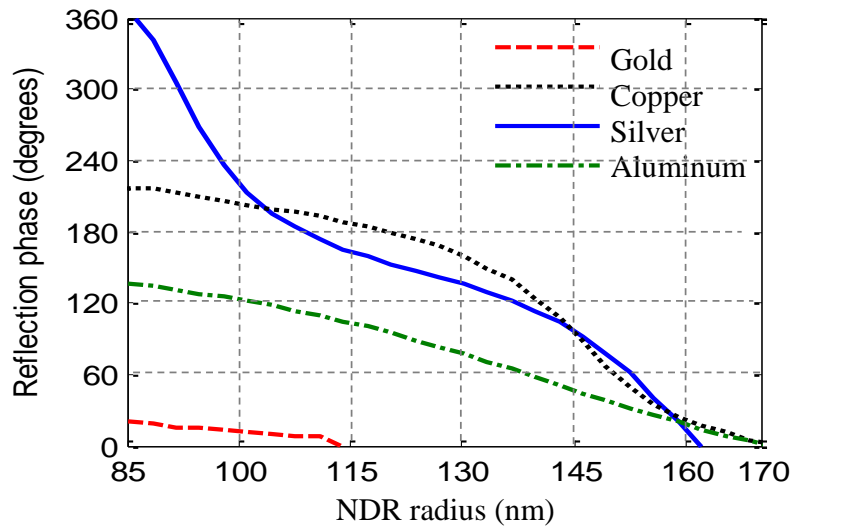

(b) Reflection coefficient phase magnitude

Figure 5 The variations of the reflection coefficient magnitude and phase versus the NDR radius at $474 \mathrm{THz}$ for the unit cell with different plane materials for $h=200 \mathrm{~nm}$, $L=350 \mathrm{~nm}$ and $h_{d}=50 \mathrm{~nm}$.

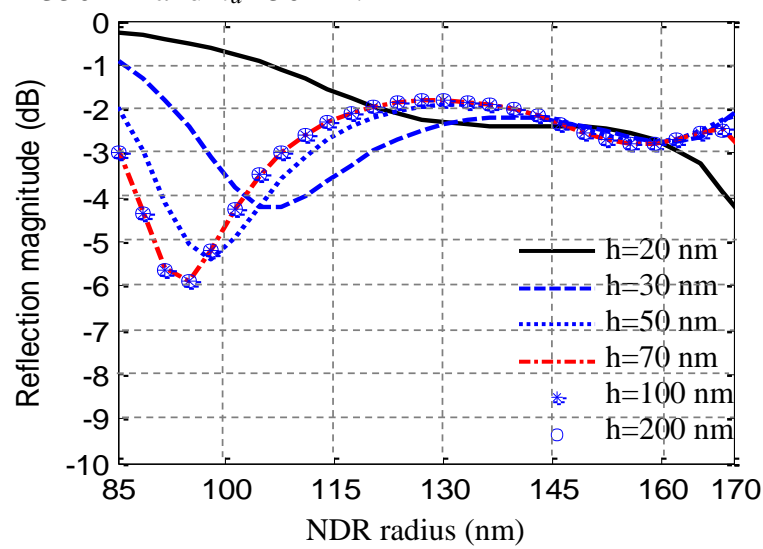

(a) Reflection coefficient magnitude

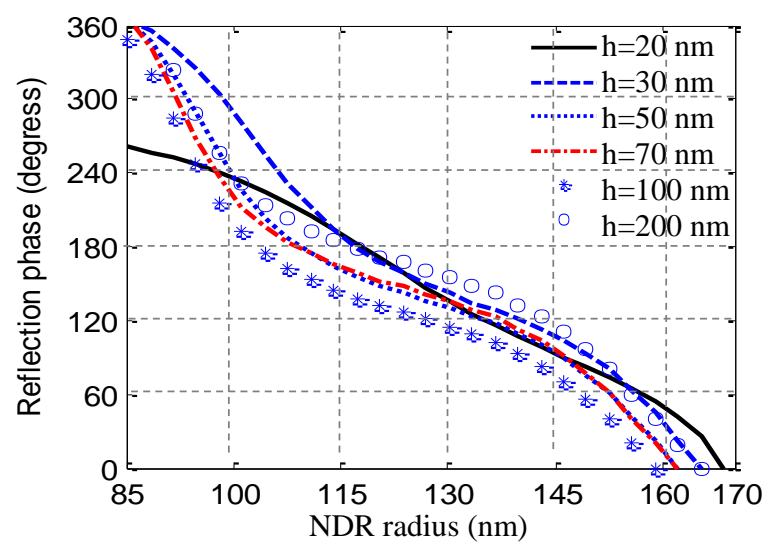

(b) Reflection coefficient phase magnitude

Figure 6 The variations of the reflection coefficient magnitude and phase versus the NDR radius of the unit cell with silver supporting plane at different plate thickness $h$, at $L=350 \mathrm{~nm}, h_{d}=50 \mathrm{~nm}$. 


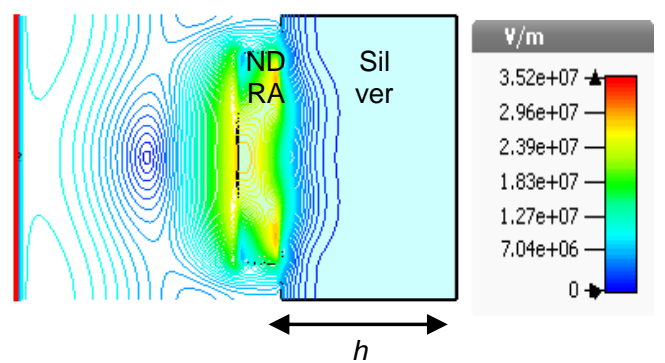

Figure 7 The electric field distribution on the unit-cell with silver supporting plane, $h=200 \mathrm{~nm}, L=350 \mathrm{~nm}, h_{d}=50 \mathrm{~nm}$ and $R=130 \mathrm{~nm}$.

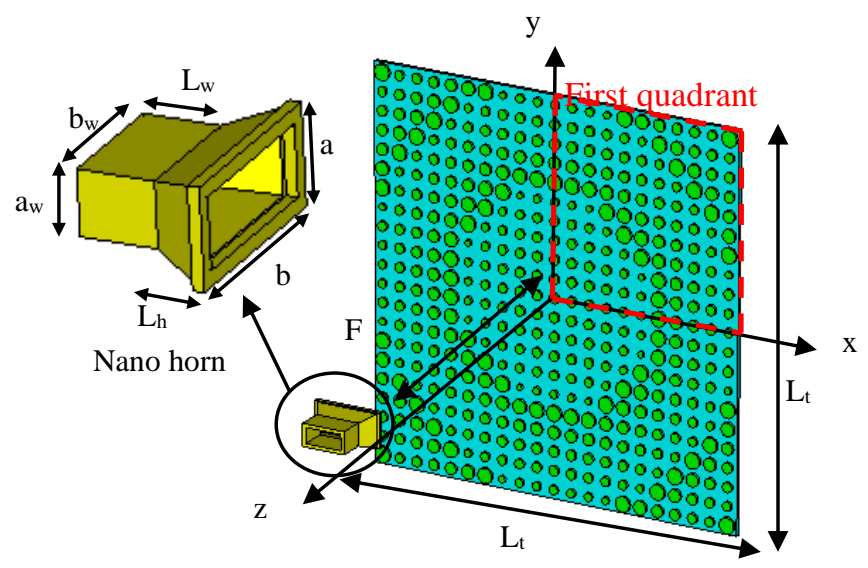

(a)

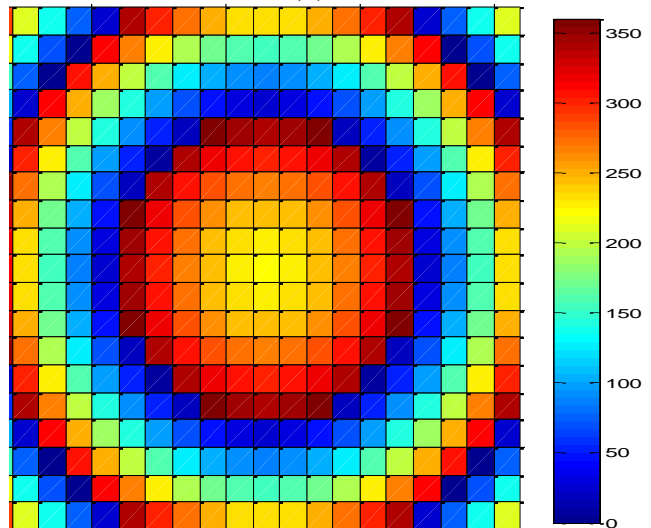

(b)

Figure 8 (a) The detailed structure of the NDRA reflectarray with silver supporting plane, (b) The phase shift distribution on the $21 \times 21$ unit-cell elements NDRA reflectarray with silver supporting plane.

A $21 \times 21$ unit cell NDRA reflectarray is simulated. Silver ground plane with $L=350 \mathrm{~nm}, h=200 \mathrm{~nm}$, and $h_{d}=50$ $\mathrm{nm}$ is considered as shown in Fig.8a. The phase distribution of the $21 \times 21$ unit cell is calculated using Eq. (7) as shown in Fig. 8 b. The array has total dimensions of $7.35 \times 7.35 \mu \mathrm{m}^{2}$. A linearly polarized pyramidal nano-horn antenna is used to feed the NDRA reflectarray located at $(0,0,8.133) \mu \mathrm{m}$ from the array aperture in the horn phase centre. The nano-horn antenna is constructed from gold with $L_{w}=487.5 \mathrm{~nm}$, aperture size $a \times b$ of $810 \mathrm{~nm} \times 1275 \mathrm{~nm}, a_{w} \times b_{w}$ of $412.5 \mathrm{~nm}$ $\times 825 \mathrm{~nm}$, and $L_{h}=470.5 \mathrm{~nm}$. The nano-horn antenna has a maximum gain of $11.1 \mathrm{~dB}$ at $474 \mathrm{THz}$. The $\mathrm{E}$ and H-plane radiation patterns at $474 \mathrm{THz}$ of the nano-horn and the NDRA reflectarray with different ground plane thickness $h=30,70$ and $200 \mathrm{~nm}$ are shown in Fig.9. For the inset variation from $-10^{\circ}$ to $10^{\circ}$, the maximum gain of the NDRA reflectarray is increased by increasing the ground plane thickness and the first side lobe level (SLL) is decreased. For $h=200 \mathrm{~nm}$ the SLL is $-15.1 \mathrm{~dB}$ and $-17.9 \mathrm{~dB}$ in E-plane and H-plane respectively, and $-23.3 \mathrm{~dB}$ in E-plane, and -14.8 $\mathrm{dB}$ in $\mathrm{H}$-plane for nano-horn respectively. The half-power beamwidth (HPBW) of the NDRA reflectarray is $4.7^{\circ}$ in Eplane and $4.5^{\circ}$ in H-plane compared to $40.3^{\circ}$ in E-plane and $41.3^{\circ}$ in $\mathrm{H}$-plane for the nano-horn antenna.

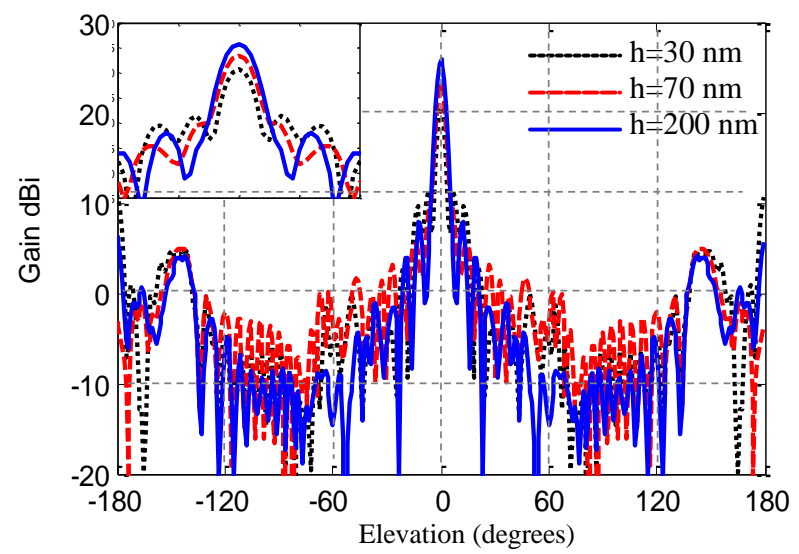

(a) E- Plane

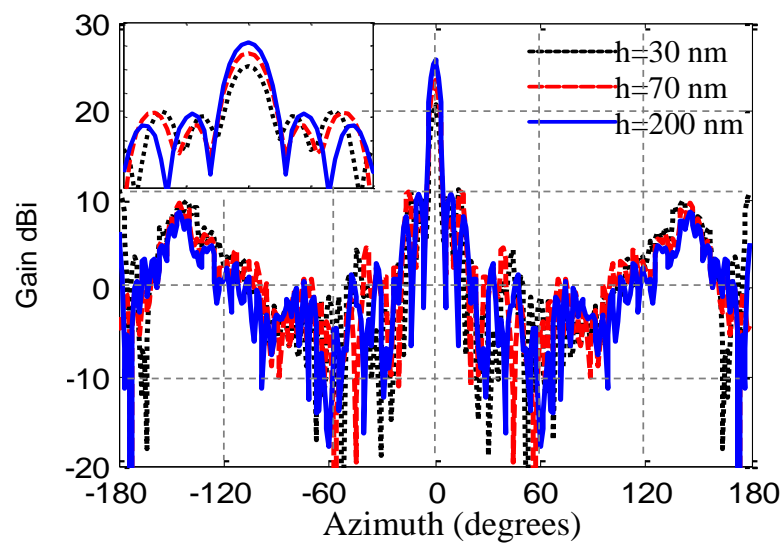

(b) H- Plane

Figure 9. The gain patterns plot for a $21 \times 21$ NDRA reflectarray for variable silver supporting plane thickness at $474 \mathrm{THz}, L=350 \mathrm{~nm}$, and $h_{d}=50 \mathrm{~nm}$.

The 3-D radiation pattern of the reflectarray at $\mathrm{f}=474$ $\mathrm{THz}$ of the NDRA reflectarray is shown in Fig.10a. The gain variations versus frequency for the NDRA reflectarray and the nano-horn are shown in Fig. 10b. The NDRA reflectarray has a maximum gain of $25.8 \mathrm{~dB}$ at $474 \mathrm{THz}$ with $1-\mathrm{dB}$ gain variation of $30 \mathrm{THz}$ (from $459 \mathrm{THz}$ to 489 $\mathrm{THz}$ ). 


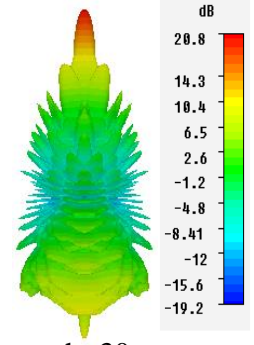

$\mathrm{h}=30 \mathrm{~nm}$

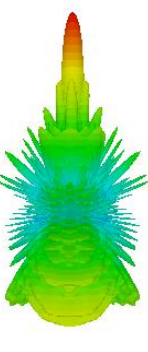

$\mathrm{h}=70 \mathrm{~nm}$

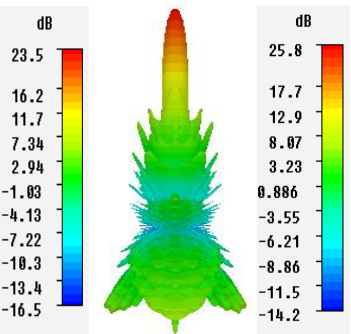

$\mathrm{h}=200 \mathrm{~nm}$ (a) The 3-D gain radiation pattern at $\mathrm{f}=474 \mathrm{THz}$

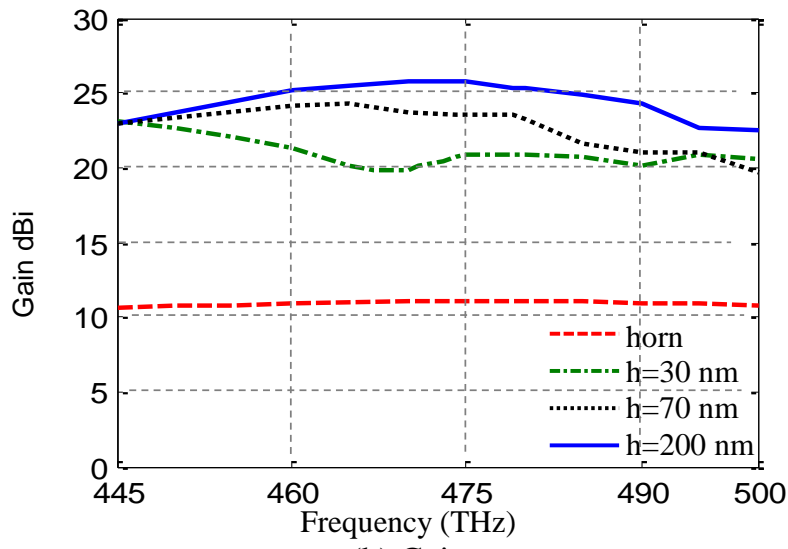

(b) Gain

Figure 10 (a) The 3-D gain radiation pattern at $f=474 \mathrm{THz}$, (b) The gain variation versus frequency for a $21 \times 21$ NDRA reflectarray at different thickness of the silver supporting plane.

\section{Design of NDRA transmitarray antenna}

The NDRA transmitarray unit-cell consists of two NDRA placed on the opposite sides of the supporting plane as shown in Fig. 11. Each NDRA has radius $R$, height $h_{d}$ and silver supporting plane with thickness $h$ and length $L$. The required compensation phase of the transmission coefficient for each unit-cell is accomplished by varying the NDR radius $\mathrm{R}$ using the wave guide simulator. Figure 12 shows the variation of the transmission coefficient magnitude and phase versus the NDR radius at $474 \mathrm{THz}$ for $L=350 \mathrm{~nm}$, $h=50 \mathrm{~nm}, h_{d}=50 \mathrm{~nm}$ for different supporting plane materials. The Aluminum supporting plane has the worst transmission coefficient variation from $-26 \mathrm{~dB}$ to $-17.3 \mathrm{~dB}$, while the silver supporting plane gives the best transmission coefficient magnitude variation from $-4 \mathrm{~dB}$ to $-2.4 \mathrm{~dB}$ for the NDR radius variation from $85 \mathrm{~nm}$ to $170 \mathrm{~nm}$. The phase of the transmission coefficient variation is $240^{\circ}$ for gold, $300^{\circ}$ for the copper, $350^{\circ}$ for the silver and $180^{\circ}$ for the Aluminum supporting plate. The silver material has the best performance for the transmitarray unit-cell with transmission coefficient magnitude variation from $-4 \mathrm{~dB}$ to $2.4 \mathrm{~dB}$ and phase variation from 0 to $350^{\circ}$. The variation of the transmission coefficient magnitude and phase versus the NDRA radius for different silver supporting plane thickness of the unit-cell is shown in Fig.13. By increasing the supporting plane thickness the transmission coefficient magnitude is decreased. The skin depth of gold is greater than that of silver at $474 \mathrm{THz}$. The skin depth of silver at $474 \mathrm{THz}$ is nearly $24.5 \mathrm{~nm}$, where the transmitarray unit-cell thickness must have approximately twice the skin depth or less to maximize the transmission from the unit-cell. A compromise between the magnitude of the transmission coefficient and phase variation has been made. The electric field distribution on the unit-cell of the NDRA transmitarray with silver supporting plane, $h=50 \mathrm{~nm}, h_{d}=50 \mathrm{~nm}$ and $R=130 \mathrm{~nm}$ is shown in (Fig. 14). The incident plane wave passes to the other side silver supporting plane. The transmission occurs as the thickness of silver supporting plane is equal to twice the silver skin depth where the transmission condition is satisfied.

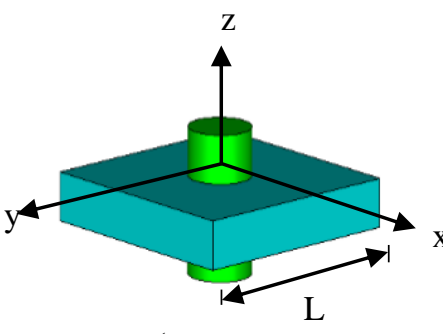

(a) 3-D view

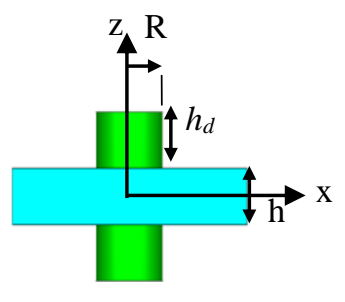

(b) Side view
Figure 11 The detailed structure of the NDRA tramsmitarray unit cell.

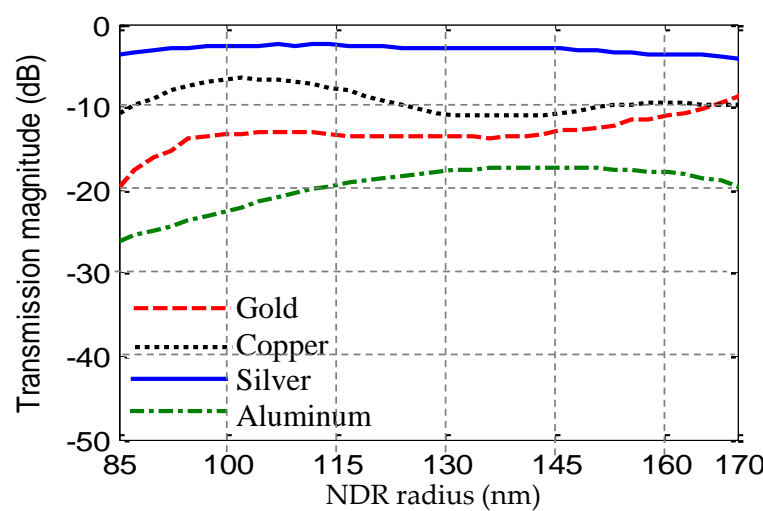

(a) Transmission coefficient magnitude

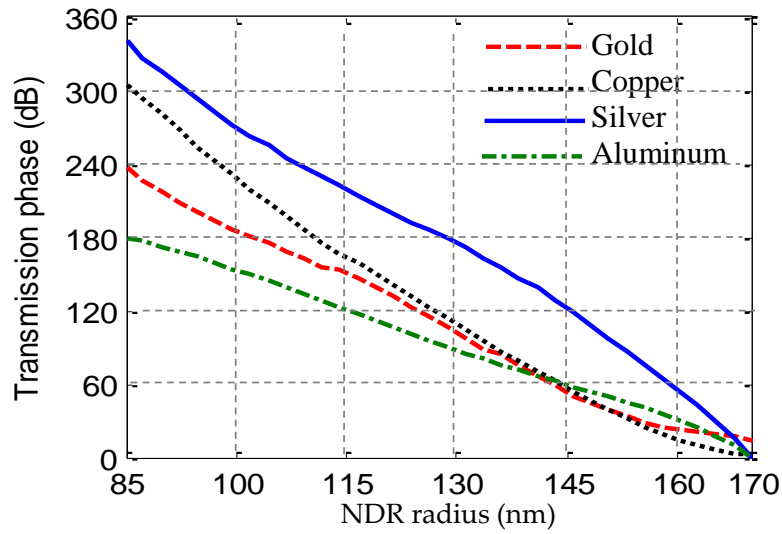

(b) Transmission coefficient phase magnitude

Figure 12 The variations of the transmission coefficient magnitude and phase of the NDR transmitarray unit cell with different supporting plane materials for $h=50 \mathrm{~nm}$, $L=350 \mathrm{~nm}$ and $h_{d}=50 \mathrm{~nm}$. 


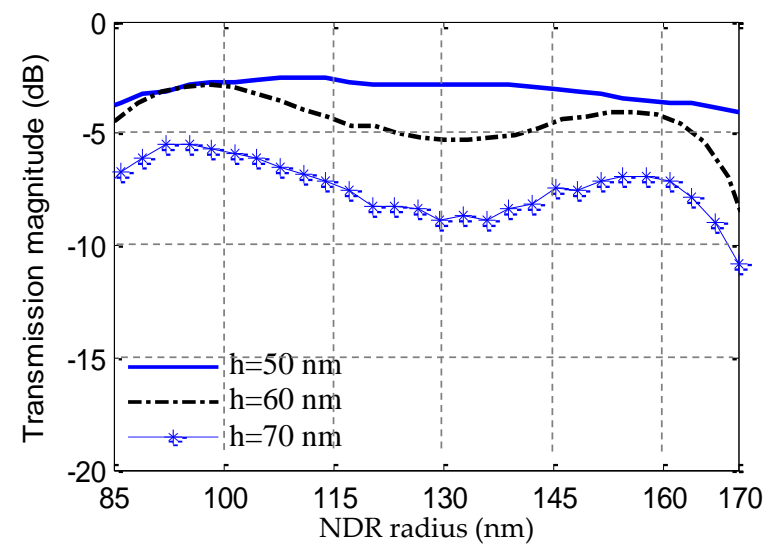

(a) Transmission coefficient magnitude

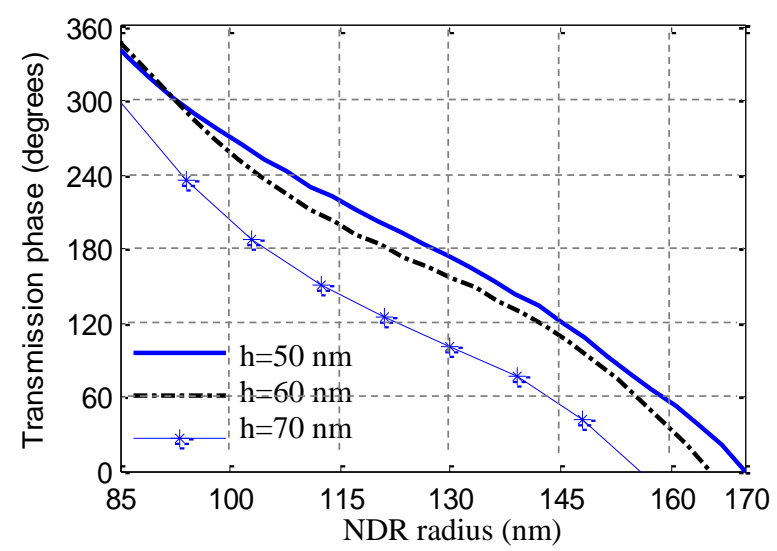

b. Transmission coefficient phase

Figure 13 The variations of the transmission coefficient magnitude and phase versus the NDR radius with silver supporting plane thickness $L=350 \mathrm{~nm}, h_{d}=50 \mathrm{~nm}$.
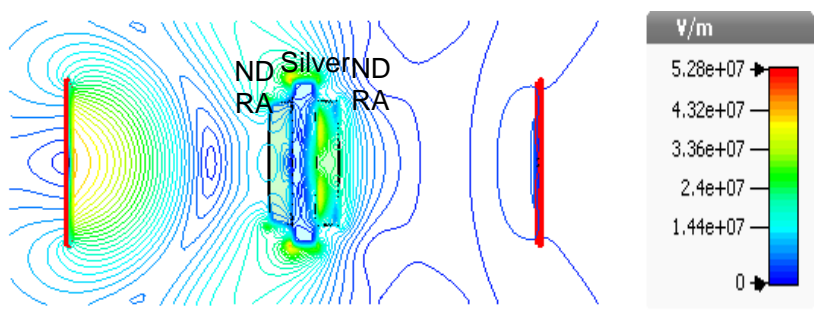

Figure 14 The electric field distribution on the NDR transmitarray unit-cell with silver supporting plane $h=50$ $\mathrm{nm}, L=350 \mathrm{~nm}, h_{d}=50 \mathrm{~nm}$ and $R=130 \mathrm{~nm}$.

A design of $17 \times 17$ unit-cells and a $21 \times 21$ unit-cells of the NDRA transmitarray at $474 \mathrm{THz}$ are investigated. The element spacing between the unit cells is $350 \mathrm{~nm}$, and supporting plane thickness $\mathrm{h}=50 \mathrm{~nm}, \mathrm{~h}_{\mathrm{d}}=50 \mathrm{~nm}$. The feeding horn is located at a distance $\mathrm{F}=8.133 \mu \mathrm{m}$ in the normal direction of the array plane. The $\mathrm{E}$ and $\mathrm{H}$-plane radiation patterns at $474 \mathrm{THz}$ for the $17 \times 17$ and the $21 \times 21$ NDRA Transmitarrays, and the horn antenna are shown in Fig. 16. For the $17 \times 17$ NDRA transmitarray, the SLL is $18.2 \mathrm{~dB}$ in $\mathrm{E}$ plane and $-14.4 \mathrm{~dB}$ in H-plane respectively. The HPBW is $6.3^{\circ}$ in E-plane and $5.8^{\circ}$ in H-plane. For the $21 \times 21$ transmitarray, the SLL for is $-18.2 \mathrm{~dB}$ in E plane and
$-15.9 \mathrm{~dB}$ in H-plane. The HPBW of the transmitarray is $5.1^{\circ}$ in E-plane and $4.8^{\circ}$ in H-plane. The 3-D radiation patterns of the transmitarrays at $\mathrm{f}=474 \mathrm{THz}$ are shown in Fig. 17a. The nano DRA transmitarray gain variation versus frequency is shown in Fig. 17b. The max gain is $23.9 \mathrm{dBi}$ for $17 \times 17$ transmitarray and $27.5 \mathrm{dBi}$ for $21 \times 21$ transmitarray and $11.2 \mathrm{dBi}$ for nano horn antenna. The $1-\mathrm{dB}$ band width is $55 \mathrm{THz}$ (from $451.5 \mathrm{THz}$ to $496.5 \mathrm{THz}$ ) for $17 \times 17$ transmitarray and $40 \mathrm{THz}$ (from $460 \mathrm{THz}$ to $500 \mathrm{THz}$ ) for $21 \times 21$ transmitarray. The E-plane and H-plane gain patterns for the $21 \times 21$ NDRA transmitarray at different frequencies are shown in Fig. 18.

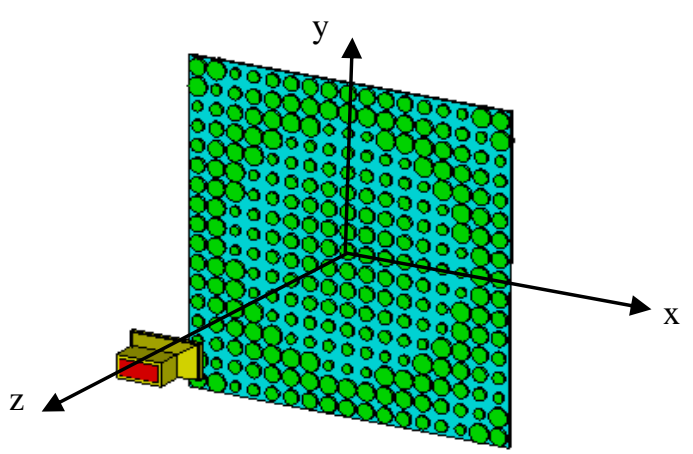

(a)

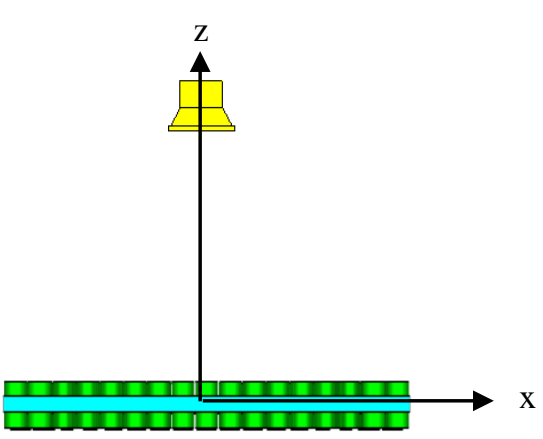

(b)

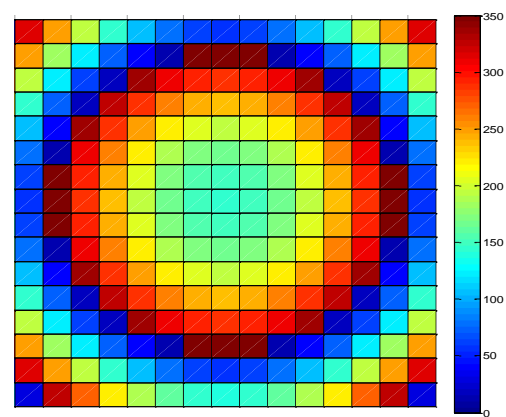

(c)

Figure 15 (a) The detailed structure of the NDRA transmitarray with silver supporting plane, (b) side view, (c) The phase shift distribution of the NDRA transmitarray with the silver supporting plane. 


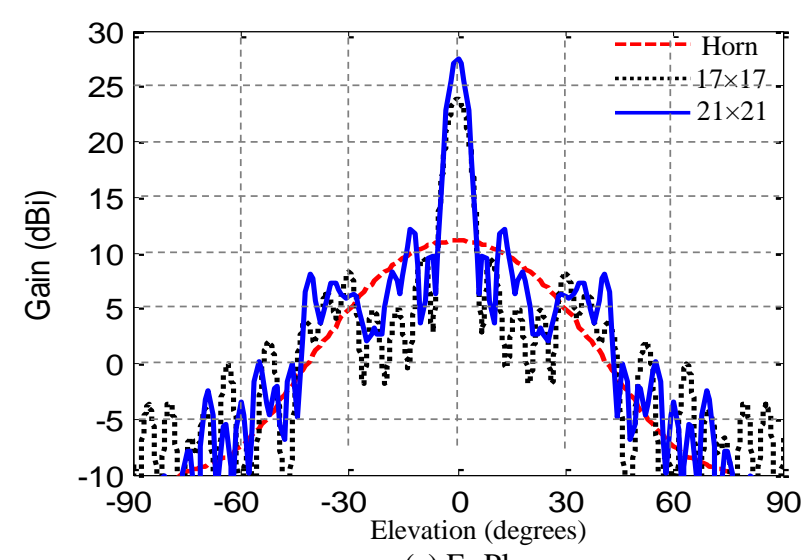

(a) E- Plane

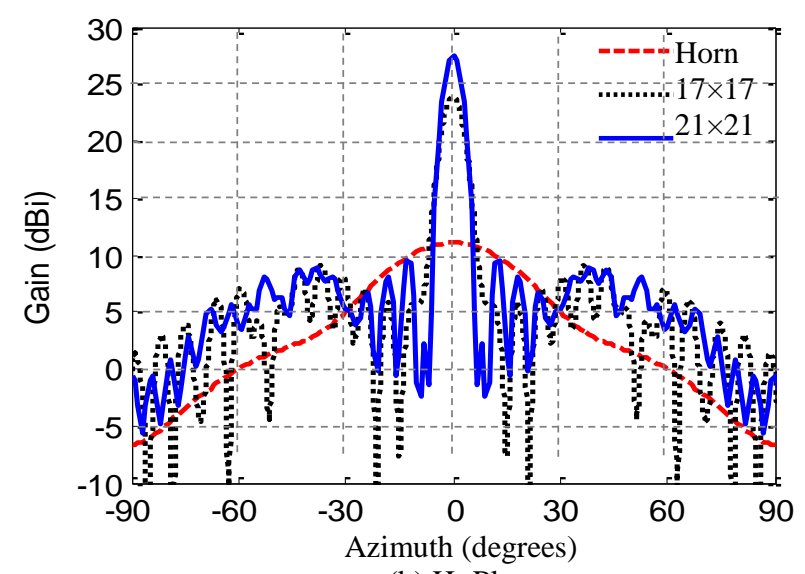

(b) H- Plane

Figure 16 The gain patterns for $21 \times 21$ NDRA transmitarray with silver supporting plane at $474 \mathrm{THz} . h=50 \mathrm{~nm}, L=350$ $\mathrm{nm}$, and $h_{d}=50 \mathrm{~nm}$.

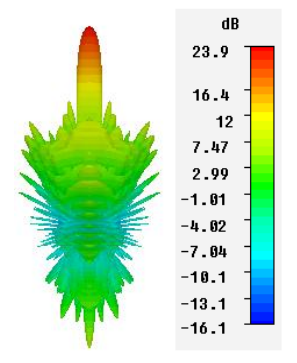

(a)
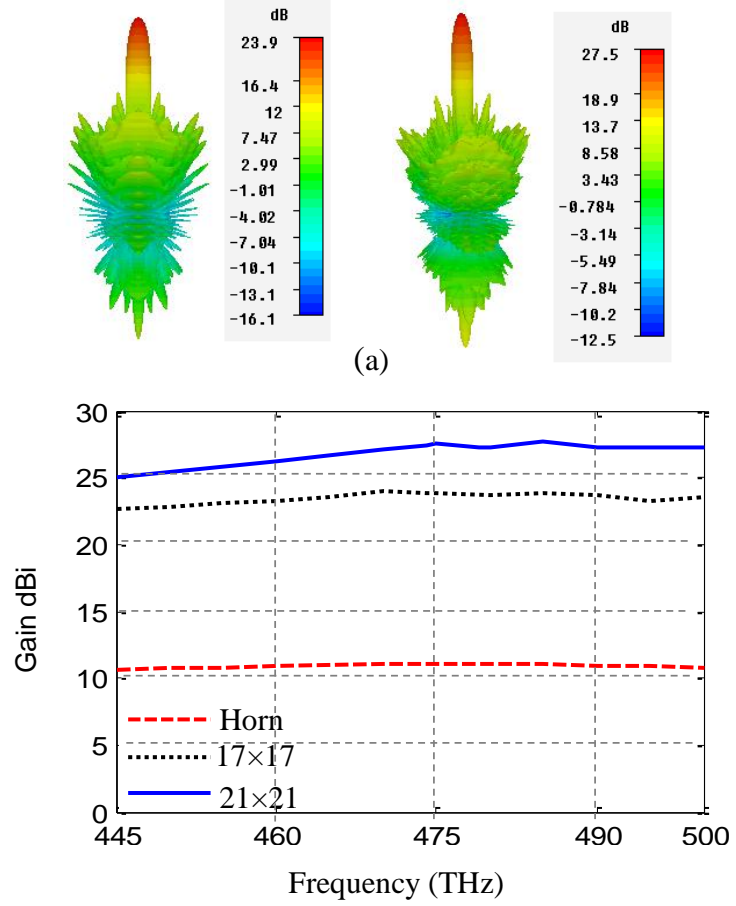

(b)

Figure 17 (a) The 3 -D gain radiation pattern at $f=474 \mathrm{THz}$. (b) The gain variation versus frequency for $17 \times 17$ and a $21 \times 21$ NDRA transmitarray with silver supporting plane.

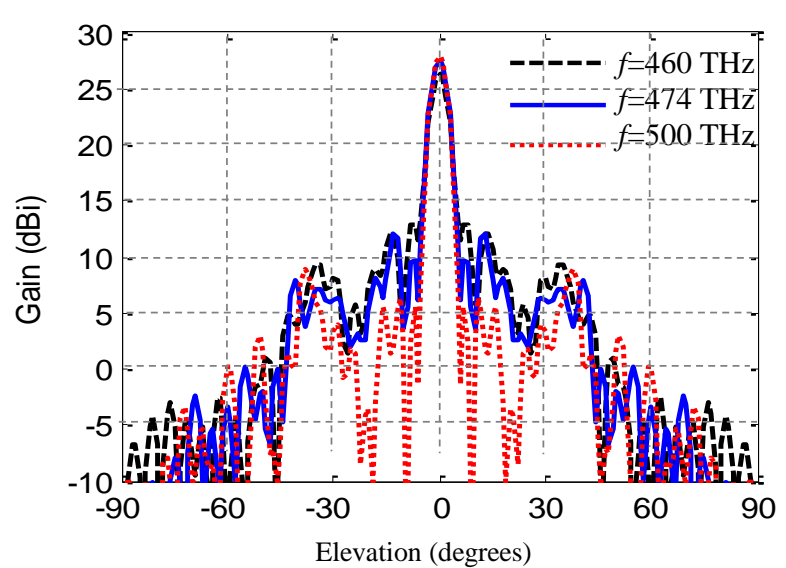

(a) E- Plane

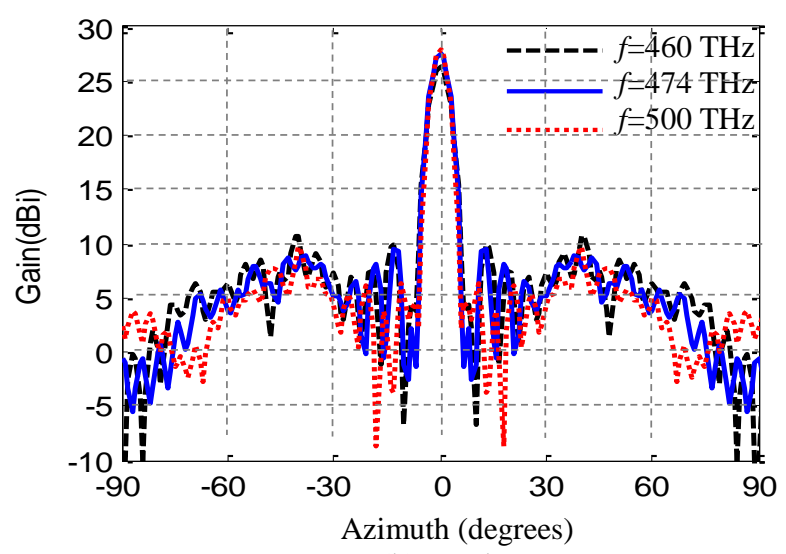

(b) H- Plane

Figure 18 The gain radiation patterns for a $21 \times 21$ NDRA transmitarray with silver supporting plane at different frequencies.

\section{Conclusion}

The use of NDR in the design of nano-reflectarray and nanotransmitarray for terahertz application at $633 \mathrm{~nm}$ is introduced. The reflectarray unit cell consists of a cylindrical NDR on a square ground plane of a good conductor. A parametric study for the unit cell dimensions optimization has been introduced. The gold ground plane has got the worst reflection coefficient (higher losses), while the silver ground plane gives the best reflection coefficient magnitude. Silver ground plane with thickness of $200 \mathrm{~nm}$ has been chosen for reflection coefficient magnitude varies from -1.7 $\mathrm{dB}$ to $-5.8 \mathrm{~dB}$ and $360^{\circ}$ phase variation. The silver ground plane with thickness $200 \mathrm{~nm}$ introduces slower phase variation and hence wider bandwidth. The NDRA reflectarray consists of $21 \times 21$ unit cells with a silver ground plane of $L=350 \mathrm{~nm}, h=200 \mathrm{~nm}$, and $h_{d}=50 \mathrm{~nm}$ is simulated using the full-wave simulator. The maximum gain of the NDRA reflectarray is increased by increasing the ground plane thickness and the SLL is decreased. The NDRA reflectarray introduces a maximum gain of $25.8 \mathrm{~dB}$ at 474 $\mathrm{THz}$ with $1-\mathrm{dB}$ gain variation bandwidth of $30 \mathrm{THz}$. The NDRA transmitarray unit-cell consists of two NDRA placed on both sides of the supporting plane, has been designed and analyzed. The Aluminum ground plane has the worst transmission coefficient variation from $-26 \mathrm{~dB}$ to $-17.3 \mathrm{~dB}$. 
The silver as a supporting plane material has shown the best characteristics when compared with gold, copper, and aluminum. Comparison between-transmitarray with $17 \times 17$ unit-cells and of $21 \times 21$ unit-cells at $474 \mathrm{THz}$ have been introduced. The maximum gain is $23.9 \mathrm{~dB}$ for the $17 \times 17$ transmitarray and $27.5 \mathrm{~dB}$ for the $21 \times 21$ transmitarray while $11.2 \mathrm{~dB}$ for the feeding nano horn antenna.

\section{References}

[1] A.C. Balanis, Antenna theory analysis and design, $3^{\text {rd }}$ Edition, John Wiley \& Sons, USA, 2012.

[2] M. Agio, "Optical antennas as nanoscale resonators," Nanoscale Journal, vol. 3, pp. 692-706, 2011.

[3] P. Muhlschlegel, H.J. Eisler, O.J.F. Martin, B. Hecht, and D. W. Pohl, "Resonant optical antennas," Science Magazine, vol. 308, pp. 1607-1609, June 2005.

[4] T.H. Taminiau, F.B. Segerink, R.J. Moerland, L. Kuipers, and N.F. Hulst, "Near-field driving of a optical monopole antenna," Journal of Optics A: Pure and Applied Optics, vol. 9, pp. 315-321, Aug. 2007.

[5] I.S. Maksymov, A.E. Miroshnichenko, Y.S. Kivshar, "Actively tunable optical Yagi-Uda nanoantenna with bistable emission characteristics," Optics express, vol. 8, pp. 89298938, Feb. 2012.

[6] H. Fischer and O.J. Martin, "Engineering the optical response of plasmonic nanoantennas," Optics express, vol. 16, no. 12, pp. 9144-9154, June 2008.

[7] R. Vajtai, Hand book of nanomaterials, Springer Handbooks, New York, USA, 2013.

[8] N. Kumar, Spontaneous emission rate enhancement using optical antennas, Ph.D. Thesis, University of California, Berkeley, USA, 2013.

[9] K.M. Luk, K.W. Leung, and J. R. James, Dielectric resonator antennas, Research Studies Press, Hertfordshire, England, 2002.

[10] L. Zou, Dielectric resonator antennas from multifunction microwave devices to optical nano-antennas, Ph.D. Thesis, School of Electrical and Electronic Engineering, University of Adelaid, Australia, March 2013.

[11] S.H. Zainud-Deen, H.A. Malhat, S.M. Gaber, M. Ibrahim, and K.H. Awadalla, "Plasma reflectarray," Plasmonics, vol. 8, no. 3, pp. 1469-1475, Sep. 2013.

[12] J. Huang, and J.A. Encinar, Reflectarray antennas, John Wiley and Sons, Inc., New Jersy, USA, 2007.

[13] S.H. Zainud-Deen, Hend A. Malhat, S.M. Gaber, and K. H. Awadalla, " Perforated Nanoantenna Reflectarray," Progress In Electromagnetics Research M, PIER M, vol. 29, pp. 253265, 2013.

[14] H.A. Malhat, S.H. Zainud-Deen, and S.M. Gaber, "Circularly Polarized Graphene Based Transmitarray for Terahertz Applications," Progress In Electromagnetics Research M, PIER M, vol. 36, pp. 185-191, 2014.

[15] R. Schumann, T. Weiland, W.H. Schilders, E.J. Maten, and S.H. Houben, "Recent advances in finite integration technique for high frequency applications," Scientific Computing in Electrical Engineering,vol.4, pp. 46-57, 2004.

[16] CST microwave studio, http://www.cst.com/

[17] M. A. Ordal, L. L. Long, R. J. Bell, S. E. Bell, R. R. Bell, R. W. Alexander, Jr., and C. A. Ward, "Optical properties of the metals $\mathrm{Al}, \mathrm{Co}, \mathrm{Cu}, \mathrm{Au}, \mathrm{Fe}, \mathrm{Pb}, \mathrm{Ni}, \mathrm{Pd}, \mathrm{Pt}, \mathrm{Ag}$, Ti, and $\mathrm{W}$ in the infrared and far infrared," APPLIED OPTICS ,vol.22, no. 7, pp.1099-11201, April 1983. 